\title{
Osteomielite criptocócica em paciente com mieloma múltiplo
}

\section{Cryptococcal osteomyelitis in a patient with multiple myeloma}

\author{
Vanessa B. Nocera ${ }^{I}$ \\ Ivo Ronchi $\mathrm{Jr}^{2}$ \\ Joelma Pietrovicz $z^{3}$ \\ Cecília N. V. Krebs ${ }^{4}$ \\ Gabriel L. Lopes ${ }^{5}$
}

\begin{abstract}
A criptococose é uma infecção fúngica sistêmica que pode acometer vários órgãos, entre eles sistema nervoso central, pulmões e ossos. A apresentação clínica mais comum deste microorganismo é a meningite, mas acometimento ósseo tem sido relatado em $5 \%$ a $10 \%$ dos casos como parte de uma infecção sistêmica. O envolvimento ósseo isolado com Cryptococcus neoformans é extremamente raro. A incidência é mais comum em pacientes imunocomprometidos, como naqueles em uso crônico de corticoterapia ou outros imunossupressores, portadores de sarcoidose, tuberculose e neoplasias. Relatamos o caso de uma paciente com mieloma múltiplo e que apresentou osteomielite por C. neoformans, sendo que não encontramos nenhum caso semelhante na literatura. O objetivo desse relato é discutir sobre a associação de criptococose em pacientes imunocomprometidos. Rev. Bras. Hematol. Hemoter. 2010;32(3):265-268.
\end{abstract}

Key words: Criptococose; infecção; osso; mieloma múltiplo; imunossupressão.

\section{Introdução}

A criptococose é uma infecção fúngica sistêmica que pode acometer vários órgãos, como pulmões, sistema nervoso central (SNC) e ossos. A apresentação clínica mais comum é meningite, mas acometimento ósseo tem sido relatado em $5 \%$ a $10 \%$ dos casos como parte de infecção sistêmica. O envolvimento ósseo isolado com Cryptococcus neoformans é extremamente raro. A incidência é mais comum em pacientes imunocomprometidos. Relatamos o caso de uma paciente com mieloma múltiplo que apresentou osteomielite isolada por $C$. neoformans, recebeu tratamento com fluconazol por 12 meses, com resolução completa do quadro. No paciente imunocomprometido, como no mieloma múltiplo, é importante considerar infecções fúngicas no diagnóstico diferencial de lesões osteolíticas ou osteogênicas. Enquanto dados radiológicos podem ajudar no diagnóstico, o acompanhamento com Raio-X não é muito útil pois a resolução ocorre lentamente. ${ }^{1}$ Manejo intensivo para diagnóstico precoce de infecções fúngicas deve ser estabelecido, incluindo análise e cultura de líquidos ou secreções, avaliações por exames de imagem e biópsias de lesões suspeitas, com o objetivo de reduzir a alta taxa de morbimortalidade das infecções criptocócicas esqueléticas. ${ }^{1}$

\section{Relato de Caso}

Paciente N.Z.R., 55 anos, feminina, em 2005 vinha apresentando há três meses febre diária, dor em antebraço direito. Tem diagnóstico de mieloma múltiplo (MM) produtor de IgA há nove anos, sendo que há dois anos está somente em acompanhamento clínico, sem sinais de atividade da doença. Ao exame físico apresentava-se em bom estado geral, com picos febris, hipocorada, com aumento de volume,

${ }^{1}$ Médica do Serviço de Hematologia do Hospital Universitário Evangélico de Curitiba (HUEC) - Curitiba-PR.

${ }^{2}$ Médico hematologista. Chefe do Serviço de Hematologia do Hospital Universitário Evangélico de Curitiba (HUEC). Chefe da Disciplina de Hematologia da Faculdade Evangélica do Paraná (FEPAR); Professor Adjunto da Disciplina de Hematologia - Curitiba-PR.

${ }^{3}$ Médica Hematologista. Serviço de Hematologia do Hospital Universitário Evangélico de Curitiba (HUEC) - Curitiba-PR.

${ }^{4}$ Médica hematologista. Serviços de Hematologia e Clínica Médica do Hospital Universitário Evangélico de Curitiba (HUEC) - Curitiba-PR.

${ }^{5}$ Acadêmico de Medicina da Faculdade Evangélica de Medicina do Paraná - Curitiba-PR.

Hospital Universitário Evangélico de Curitiba-PR.

Correspondência: Vanessa B. Nocera

Inter Rad, Rua Gastão Câmara, 242 - Bairro Bigorrilho

80730-300 - Curitiba-PR - Brasil

Tels.: (55 41)3024-7070

E-mail:vanessabaldissera@hotmail.com

Doi:10.1590/S1516-84842010005000081 
calor local e dor à palpação em região lateral de antebraço direito, numa área endurecida de aproximadamente $5 \mathrm{~cm}$ de diâmetro. $\mathrm{O}$ hemograma revelou anemia normocrômica e normocítica $(\mathrm{Hb}=9,2 \mathrm{~g} / \mathrm{dL})$, leucócitos e plaquetas normais, VHS $=102 \mathrm{~mm}$, e dosagem de IgA normais. Aspirado de medula óssea hipocelular, com $1 \%$ de plasmócitos. Raio-X de tórax normal; raio-X de antebraço: lesão osteolítica de região proximal de rádio direito, com limites evidentes e sem esclerose na ulna proximal. Foi coletado material purulento da lesão de antebraço por aspirado com agulha fina, com culturas para bactérias, BAAR, baciloscopias de Ziehl-Gabbet e ZiehlNielsen negativas. As hemoculturas também foram negativas. Realizou-se tratamento empírico com ceftriaxona e vancomicina por 18 dias, com resultado insatisfatório. Foi feita então uma biópsia a céu aberto da lesão, com achados compatíveis com Cryptococcus sp (Figuras 1 e 2). Iniciou-se terapia com anfotericina lipossomal, sendo necessária a sua substituição

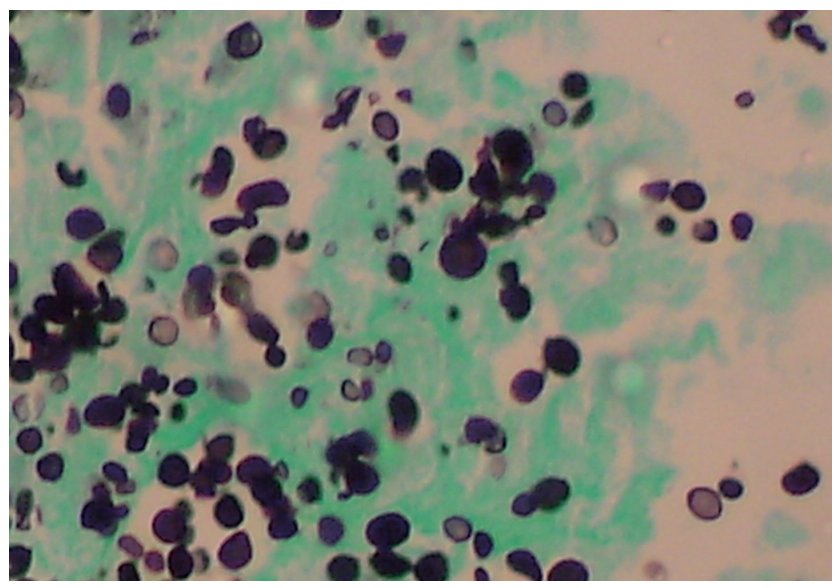

Figura 1. Tecido corado em Grocott, mostrando fungos encapsulados (Cryptococcus) (aumento 1000x)

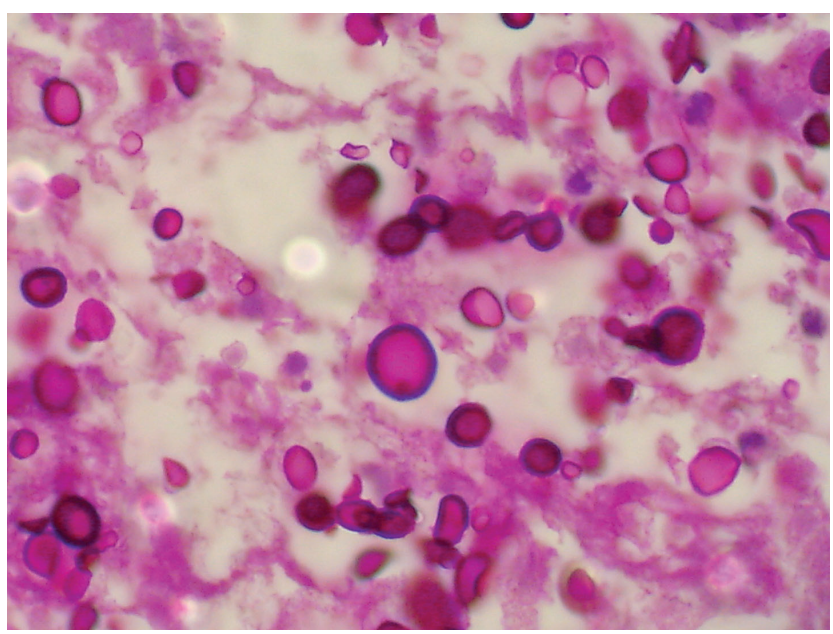

Figura 2. Numerosas estruturas encapsuladas (cryptococcus), através de coloração em PAS (aumento de 1000x) por fluconazol, pelos efeitos colaterais, o qual foi usado por cerca de seis meses, e, após, dose de manutenção até completar 12 meses, por ter apresentado resposta clínica lenta. Evoluiu com resolução clínica satisfatória e normalização do VHS e Rx de controle.

\section{Discussão}

A criptococose é uma micose sistêmica, causada pelo Cryptococcus neoformans, um fungo leveduriforme, encapsulado, de reprodução por brotamento, encontrado em todo o mundo, principalmente em solos contaminados com dejetos de pombos e outras aves. ${ }^{2,3}$ Acomete com maior frequência o SNC, os pulmões e, menos comumente, sangue, pele, ossos, articulações e próstata. ${ }^{1,2,4,5}$ Não há predileção óbvia por idade, sexo ou profissão. Ocorre preferencialmente em pacientes com algum grau de imunodeficiência, com destaque para a Síndrome da Imunodeficiência Adquirida, ${ }^{3}$ com associação de mais de $80 \% .{ }^{6}$ Outras condições relacionadas são: sarcoidose, ${ }^{3,7}$ tuberculose, uso crônico de corticosteroides, ${ }^{5}$ processos linfoproliferativos malignos, transplante de órgãos e diabetes. ${ }^{4,6,8}$ Pacientes imunocompetentes raramente são atingidos. ${ }^{8}$ Não encontramos nenhum relato de caso de osteomielite criptocócica em pacientes com mieloma múltiplo durante revisão da literatura.

A inalação de esporos aerossolizados do C. neoformans é considerada a via primária de aquisição. Dependendo da condição imunológica do paciente, a infecção pode se resolver espontaneamente ou sofrer disseminação hematogênica com comprometimento de outros órgãos. ${ }^{5} \mathrm{O}$ envolvimento ósseo ocorre em 5\% a $10 \%$ dos casos de infecção disseminada, porém a osteomielite isolada é extremamente rara, ${ }^{1,3,5,8,9}$ sendo a coluna vertebral o local mais comumente acometido, seguido de pelve e costela. ${ }^{10}$ Lesão em um sítio único é a regra. ${ }^{3}$ Acredita-se que a osteomielite isolada seja proveniente da disseminação hematogênica originária de um foco pulmonar autolimitado e assintomático. ${ }^{1,4}$ Pode ocorrer doença extrapulmonar, na ausência de comprometimento pulmonar aparente. Curiosamente, pacientes com osteomielite criptococócica são geralmente afetados por condições imunossupressoras menos severas que naqueles com meningite ou pneumonia. ${ }^{7}$

O Cryptococcus neoformans não é altamente patogênico. Os três principais fatores de virulência são a cápsula de complexo polissacarídeo, produção do pigmento de melanina (proteção contra estresse oxidativo) e a habilidade em crescer em $37^{\circ} \mathrm{C},{ }^{2,6} \mathrm{~A}$ cápsula é o fator de virulência mais importante, podendo ser imunossupressor, limitar a produção de óxido nítrico, interferir nos processos de apresentação de antígenos e proporcionar ao microorganismo propriedades antifagocíticas. Por esta razão, a imunidade humoral exerce uma função importante para a defesa do hospedeiro, diferenciando-se do que ocorre nas demais 
infecções fúngicas, nas quais a imunidade celular é a pedra angular. $^{2}$

No MM ocorre uma proliferação clonal de plasmócitos que produzem uma proteína monoclonal. Vários fatores podem ser responsáveis pela suscetibilidade aumentada dos pacientes com mieloma a infecções: concentração diminuída de imunoglobulinas normais, capacidade de edificar uma resposta imune primária gravemente deprimida, caracterizada por um tempo prolongado com títulos máximos diminuídos de anticorpos, e um declínio rápido nas concentrações de anticorpos. ${ }^{11}$

Os sintomas predominantes na apresentação da osteomielite criptocócica são: dor, aumento de volume e incapacidade funcional do local acometido. ${ }^{1,3} \mathrm{O}$ paciente geralmente permanece afebril e em bom estado geral durante o curso da doença. ${ }^{3}$ A evolução costuma ser arrastada, com média de três meses entre o surgimento dos sintomas e a procura de atendimento médico. ${ }^{1}$

O hemograma normalmente está sem alterações ${ }^{3} \mathrm{e}$ a velocidade de hemossedimentação aumentada na maioria dos casos. As alterações radiológicas são inespecíficas, sendo mais frequente lesão lítica ou erosiva, com pouca ou nenhuma reação periosteal. ${ }^{12}$ Lesão infiltrativa, com extensão para ossos adjacentes, colapso vertebral e áreas de fratura patológica também podem ser vistas. ${ }^{1,3}$ Os achados radiológicos geralmente são bastante similares àqueles encontrados na tuberculose óssea, na qual se torna um diagnóstico diferencial relevante a ser excluído. ${ }^{2}$

Com exceção do acometimento do SNC, na maioria dos casos é difícil estabelecer o diagnóstico de criptococose tecidual. Devem-se obter hemoculturas e amostras do tecido para cultura e estudo histopatológico, através de biópsia excisional ou punção aspirativa da lesão. Como diagnóstico complementar pode ser feita a pesquisa de antígenos criptocócicos séricos que, apesar de algumas vezes ser negativa, alguns estudos defendem que sua presença é sinal de melhor prognóstico e resposta terapêutica. ${ }^{6}$

O diagnóstico diferencial deve ser feito com outras infecções fúngicas, Mycobacterium tuberculosis, micobactérias atípicas e brucelose. Tumores ósseos metastáticos e, principalmente, os primários, têm por característica as lesões líticas com acometimento do periósteo. ${ }^{10}$

$\mathrm{O}$ tratamento ainda não segue um protocolo bem estabelecido. A escolha e o tempo do tratamento dependem tanto do sítio anatômico acometido quanto da imunidade do paciente. Pacientes imunocomprometidos com doença pulmonar ou extrapulmonar devem ser tratados como aqueles com comprometimento do SNC. ${ }^{13}$

As opções utilizadas atualmente são uma combinação de anfotericina B endovenosa $(0,5 \mathrm{mg}$ a $1,0 \mathrm{mg} / \mathrm{kg} / \mathrm{d}$ por seis a dez semanas, com dose total de $1 \mathrm{~g}$ a $2 \mathrm{~g}$ ) associada a 5 -fluocitosina via oral $(100 \mathrm{mg} / \mathrm{kg} / \mathrm{d})$. Estudos têm mostrado a eficácia do fluconazol (200 mg a $400 \mathrm{mg} / \mathrm{d}$ via oral), por três a seis meses, assim como itraconazol, cetoconazol e posaconazol, ${ }^{2,13-16}$ sendo que, destes, o fluconazol permanece como primeira escolha. A associação de anfotericina B com fluconazol mostrou eficácia superior ao uso dessas drogas isoladamente. ${ }^{15,16}$

Pelo pequeno número de casos de osteomielite criptocócica descritos, ainda não há consenso na duração da terapia antifúngica, mas recomenda-se tratamento mínimo de três meses. Em casos de acometimento extrapulmonar, exceto SNC, a resolução dos sinais e sintomas ou outras marcas da doença indicam a duração do tratamento. ${ }^{13}$ Em muitos casos torna-se necessária a extensão da terapia com fluconazol por um período mais prolongado ("terapia de consolidação"). Também não há consenso sobre manutenção da terapia antifúngica como profilaxia nos pacientes imunodeprimidos após o tratamento da osteomielite criptocócica. Em muitos casos, a intervenção cirúrgica com drenagem e desbridamento é necessária, podendo ocorrer resolução da infecção apenas com estes procedimentos, sem necessidade de terapia antifúngica adjuvante. ${ }^{1,9,13}$

Em todos os casos é imperativo cuidadosa avaliação do SNC para afastar meningite oculta concomitante. ${ }^{13} \mathrm{O}$ rápido diagnóstico e apropriado tratamento reduz a morbidade e previne a progressão para um potencial acometimento do SNC..$^{13}$

\begin{abstract}
Cryptococcosis is a systemic fungal infection that can involve several organs, including the central nervous system, lungs and bones. The most common clinical presentation of this microorganism is meningitis however bone involvement has been reported in 5 to $10 \%$ of cases of systemic infections. Bone infections caused by Cryptococcus neoformans without other associated infections are extremely rare. The incidence is more common in immunocompromised patients, for example individuals on immunotherapy using corticosteroids or other immunosuppressants and those with sarcoidosis, tuberculosis and other malignancies. Here we report the case of a patient with multiple myeloma who presented with osteomyelitis due to C. neoformans: no other similar cases were found in the literature. The purpose of this report is to discuss the association of cryptococcosis in immunocompromised patients. Rev. Bras. Hematol. Hemoter. 2010;32(3):265-268.
\end{abstract}

Key words: Cryptococcosis; infection; bone; multiple myeloma; immunosuppression.

\section{Agradecimentos}

Agradecemos aos seguintes profissionais pelo grande auxílio dispensado: Dr. Manuel Ruedas Guerrero (Ortopedia), Dr. Afonso Coelho (Patologia), Dr. Sérgio Ricardo Penteado Filho (Infectologia), Dra. Lisiane Cristine da Mota Cabral (Patologia). 


\section{Referências Bibliográficas}

1. Behrman RE, Masci JR, Nicholas P. Cryptococcal skeletal infections: case report and review. Rev Infect Dis. 1990;12 (2):181-90

2. Murphy SN, Parnell N. Fluconazole treatment of cryptococcal rib osteomyelitis in an HIV-negative man. A case report and review of the literature. J Infect. 2005;51(5):309-11.

3. Wildstein MS, Martin SM Jr, Glaser JA. Cryptococcal osteomyelitis in a 20-year-old male with sarcoidosis. Spine J. 2005;5(4): 467-70.

4. Matsushita T, Suzuki K. Spastic paraparesis due to cryptococcal osteomyelitis. A case report. Clin Orthop Relat Res. 1985;196: 279-84.

5. Chang WC, Tzao C, Hsu HH. Isolated cryptococcal thoracic empyema with osteomyelitis of the rib in an immunocompetent host. J Infect. 2005;51(3):117-19.

6. Chayakulkeeree M, Perfect JR. Cryptococcosis. Infect Dis Clin North Am. 2006;20(3):507-44.

7. Kumlin U, Elmqvist LG, Granlund M, Olsen B, Tärnvik A. CD4 lymphopenia in a patient with cryptococcal osteomyelitis. Scand J Infect Dis. 1997;29(2):205-06.

8. Govender S, Mutasa E, Parbhoo AH. Cryptococcal osteomyelitis of the spine. J Bone Joint Surg Br. 1999;81(3):459-61.

9. Govender S, Ganpath V, Charles RW, Cooper K. Localized osseous cryptococcal infection. Report of 2 cases. Acta Orthop Scand. 1988;59(6):720-2.

10. Su CT, Chen LK, Tsai YF, Kuo CJ, Lu CL, Chen HY. Disseminated cryptococcosis with pulmonary and marrow involvement mimicking radiological features of malignancy. J Chin Med Assoc. 2004;67(2):89-92.

11. Foerster J. Multiple Myeloma. In: Lee GR, Bithell TC, Foerster J, et al. Wintrobe Hematolog Clín, São Paulo: Manole; 1998. p. 2444-2478

12. Goldshteyn N, Zanchi A, Cooke K, Agha R. Cryptococcal osteomyelitis of the humeral head initially diagnosed as avascular necrosis. South Med J. 2006;99(10):1140-1
13. Saag MS, Graybill RJ, Larsen RA. Practice guidelines for the management of cryptococcal disease. Clin Infect Dis. 2000;30: $710-8$.

14. Gumbo T, Hakim JG, Mielke J. Cryptococcus myelitis: atypical presentation of a common infection. Clin Infect Dis. 2001;32 (8): $1235-6$.

15. Rachwalski EJ, Wieczorkiewicz JT, Scheetz MH. Posaconazole: an oral triazole with an extended spectrum of activit. Ann Pharmacother. 2008;42(10):1429-38.

16. Kwon DS, Mylonakis E. Posaconazole: a new broad-spectrum antifungal agent. Expert Opin Pharmacother. 2007;8(8): $1167-78$.

17. Liu PY. Cryptococcal osteomyelitis: Case report and review. Diagn Microbiol Infect Dis. 1998;30(1):33-5.

18. Sorensen RU, Boehm KD, Kaplan D. Cryptococcal osteomyelitis and cellular immunodeficiency associated with interleukin-2 deficiency. J Pediatr. 1992;121(6):873-9.

19. Jarvis JN, Dromer F, Harrison TS, Lortholary O. Managing cryptococcosis in the immunocompromised host. Curr Opin Infect Dis. 2008;21(6):596-603.

20. Tawfiq JAA, Ghandour J. Case Report: Cryptococcus neoformans abscess and osteomyelitis in an immunocompetent patient with tuberculous lymphadenitis. Infection. 2007;35(5):377-82.

Avaliação: Editor e dois revisores externos Conflito de interesse: sem conflito de interesse

Recebido: 26/07/2009

Aceito: 07/12/2009 